\title{
Measuring the impact of certified electronic health record technology on cost, quality and safety outcomes
}

\section{Joseph G. Conte* and Anyi Chen}

Staten Island PPS,

1 Edgewater Plaza - Suite 700,

Staten Island, NY 10305, USA

Email: Jconte@statenislandpps.org

Email: Achen@statenislandpps.org

*Corresponding author

\section{Terrence Cahill, Ning J. Zhang, Fortunato Battaglia}

\author{
Department of Medical Sciences, Neurology and Psychiatry, \\ Hackensack Meridian School of Medicine, \\ Seton Hall University, \\ 340 Kingsland Street - Building123, \\ Room 4413, Nutley, NJ 07110, USA \\ Email: Terrence.Cahill@shu.edu \\ Email: Ning.Zhang@shu.edu \\ Email: Fortunato.battaglia@shu.edu
}

\section{Kenneth R. Ong}

\author{
New York, USA \\ Email: kennethrong@gmail.com
}

\begin{abstract}
Fewer than 9\% of US hospitals had basic EHR systems as of 2008 . The HITECH Act funded a multi-billion-dollar investment to close this gap requiring standards known as meaningful use (MU). The study was undertaken to assess whether this investment achieved the stated aims of MU; EHR adoption, lower cost and higher quality. The study is across-sectional, retrospective design; it employed two cohorts, MU vs. non-MU hospitals. Publicly reported, risk adjusted data from 4,221 hospitals (95\%) on clinical, cost and safety metrics were analysed. The findings: EHR adoption by hospitals responded positively in response to incentives, rising from $8 \%$ to $55 \%$. Quality outcomes showed 21,000 fewer deaths $(\mathrm{p}<0.05)$ between the three clinical conditions (heart attack, heart failure, pneumonia). The HITECH Act had a positive return on investment with lower hospital cost per discharge of \$327 $(p<0.05)$. The study results suggest hospitals with EHRs' that met MU standards demonstrate statistically significant mortality and cost improvement.
\end{abstract}

Keywords: meaningful use; HITECH Act; certified electronic health records; clinical outcomes; cost per discharge; EHR adoption; quality outcomes; healthcare information technology; HIT. 
Reference to this paper should be made as follows: Conte, J.G., Chen, A., Cahill, T., Zhang, N.J., Battaglia, F. and Ong, K.R. (2019) 'Measuring the impact of certified electronic health record technology on cost, quality and safety outcomes', Int. J. Computational Medicine and Healthcare, Vol. 1, No. 1 , pp.88-100.

Biographical notes: Joseph G. Conte is currently the Executive Director of the Staten Island PPS tasked with carrying out the New York Medicaid Redesign Waiver. He also leads the National Collaborative Consortium for Substance Use Disorder. Immediately prior to the PPS, he was the Sr. V.P. for Administration at Richmond University Medical Center. He also served as the Exec. V.P. at Catholic Health Service of Long Island and Sr. V.P. of Quality at the NorthWell Health System following time at St. Luke's/Roosevelt Hospital and Beth Israel Medical Center. He has served as an Adjunct Professor at Columbia University Mailman School of Health and Wagner College School of Business Administration.

Anyi Chen is the Chief Information Officer at Staten Island Performing Provider System. She provides leadership for the creation of an innovative population health platform to support New York and Texas Medicaid Redesign projects. With over ten years of experience working in the hospital and healthcare industry, She specialises in Healthcare Information Technology (HIT), Digital Transformation, Informatics, Data Visualisation, Advanced Data Warehousing and Business Analytics. She is an innovative leader who has helped healthcare organisations turn complex healthcare data into actionable insights and succeed in the transition to value-based care. Prior to Staten Island PPS, she was the Director of Clinical Data Analytics at The Mount Sinai Hospital.

Terrence Cahill is the Department Chair and Associate Professor at Department of Interprofessional Health Sciences and Health Administration of Seton Hall University. His academic career follows an executive career in which he served as the CEO and COO of a N.J. community hospital, Senior VP for a regional managed care organisation, and, Regional VP for a national health care insurer. $\mathrm{He}$ is a Fellow of the American College of Health Care Executives. His research interests include health care leadership, particularly related to the top management team, organisational sense making, the development of strategic thinking capability, and how generational differences are affecting organisational performance in health care organisations.

Ning J. Zhang started his faculty career in the Doctoral Program in Public Affairs in the University of Central Florida, Orlando, FL after graduating from the Department of Health Administration with a $\mathrm{PhD}$ in 2003. He received tenured in 2009 and continued to teach as an Associate Professor till 2014 when he moved to Seton Hall University, South Orange, NJ. At SHU, he was promoted to a Full Professor in 2014 and the Associated Dean for Academic Affairs in 2018. He became the Editor-in-Chief of the International Journal of Computational Medicine and Healthcare in 2018.

Fortunato Battaglia is a Professor of Medical Sciences and Director of the Neuroscience and Behavior at the Hackensack Meridian School of Medicine at Seton Hall University. He received his $\mathrm{MD}$ and $\mathrm{PhD}$ from the University of Messina, Italy. He continued his post-doctoral training at the National Institutes of Health, New York University, and Columbia University. His laboratory uses transcranial magnetic stimulation, transcranial direct current stimulation, and high-density electroencephalography as tools for brain research and therapeutic intervention in neurology and psychiatry. Currently, the focus of his research is 
Integrative Medicine. He is investigating the effect of complementary therapies and nutrition on cortical plasticity and their integration with conventional treatments.

Kenneth R. Ong was the Chief Medical Informatics Officer of NewYork-Presbyterian/Queens, former VP of Medical Informatics of the Catholic Health Services of LI, former DMI of North Shore LIJ Health System and St. Vincent CMC. The third edition of his award winning 'Medical Informatics: an Executive Primer' was released at the 2015 HIMSS Conference. The Modern Healthcare named him as one of the country's top 25 clinical informaticists in 2012. He is a recipient of the AMDIS Award in Applied Medical Informatics and the CDC's Charles C. Shepard Science Award. He is a former deputy commissioner in the New York City Department of Health and Mental Hygiene.

\section{Introduction}

As late as 2008, fewer than 9\% of hospitals had basic EHR systems (Institute of Medicine, 2001; Desroches et al., 2013; Jha et al., 2009). The Health Information Technology for Economic and Clinical Health Act (HITECH) legislation of 2008 was created to stimulate the adoption of health information technology. This multi-billion dollar investment, coupled with excitement generated by the informatics literature (Hillestad et al., 2005) that suggested that nearly $\$ 80$ billion in savings would accrue annually from EHR adoption nationwide spurred a rush to EHR adoption (Desroches et al., 2013). This level of investment and legislation (the HITECH legislation) that fundamentally transforms reimbursement models and imposes penalties for non-adoption, warrants a careful examination with respect to return on investment and clinical outcomes.

The HITECH Act legislation while bold and strategic, was designed without empirical evidence that the investment would yield improved outcomes in quality, cost and safety. The belief that EHR adoption represents a significant component of the solution to control the cost trajectory of healthcare and solve the nation's quality and safety rests on conflicting research (Himmelstein et al., 2009; DesRoches et al., 2010). At present the evidence is at best inconclusive and dependent on measurement parameters, time periods included and available survey data (Hillestad et al., 2005; Bunti et al., 2011; Blumenthal and Tavenner, 2010).

The US healthcare system is under enormous pressure to increase quality, value and bend the three trillion dollar cost of care trajectory. The adoption of payment reform paradigms such as value-based purchasing (VBP), MACRA and Medicare PQRI outcome measures focusing on quality and cost are the key indicators to assess improvement and progress (VanLare and Conway, 2012; McHugh and Joshi, 2010; Ranawat et al., 2009) and the role of EHRs remains a focus of attention. However, most prior studies analysing the impact of EHRs' focused solely on process measures for quality measurement. These measures assess compliance with steps in care protocols, while a proxy assessment of quality (Donabedian, 1998; Donabedian, 2005; Mant, 2001) they do not align with current VBP models where measurable outcomes linked to expenditure such as readmission, cost per discharge, infection and mortality rates drive reimbursement 
schema. Cost calculations were not standardised and therefore not comparable from study to study (Himmelstein et al., 2009; DesRoches et al., 2010; Chaudry et al., 2006; Appari et al., 2012; Agha, 2014). This study includes publicly reported and risk adjusted, safety, quality outcomes as well as cost per discharge measurements to assess whether MU adoption of EHR systems makes a difference in these outcomes. VBP links payment directly to the quality of care provided, it moves the focus from volume to value (Porter, 2006).

\section{Methods}

\subsection{Study design}

The study is a cross-sectional, retrospective design; it employs two cohorts, MU vs. non-MU hospitals. Publicly reported data on clinical outcomes, cost and safety from 4,221 (or 95\%) of the nation's hospitals were included in the analysis to identify if there is a difference in outcomes between the hospital cohorts.

\subsection{Data sources}

The analytic sample was comprised of 4,221 non-federal US acute care hospitals using data reported from 2011 through 2013. Data was drawn from three publicly reported national databases with respect to hospital's technology status, costs and performance on publicly reported clinical outcomes, functional characteristics and demographics.

These databases are the only source utilised by CMS for reimbursement and public reporting purposes when determining which organisation had achieved EHR implementation that meets MU standards, risk adjusted quality outcomes and identification of standardised costs controlling for multiple variables. The majority of data utilised on the previous assessment of EHR adoption and its impact on quality, safety and cost by other large national studies (Himmelstein et al., 2009; Appari et al., 2012; Agha, 2014; Ding and Xiaoseng, 2011; Jones et al., 2014), relied either on self-reported survey data, non-risk adjusted clinical performance or cost report data that was not standardised for multiple hospital specific or regional variables.

The MU data file is the most current and accurate database of hospitals attesting to and being verified as meeting meaningful use (MU) standards as well as payments being issued based on performance validation. This current study includes a sample of 4,221 hospitals (or 95\%) of hospitals nationwide. In comparison, the American Hospital Association 2012 annual survey of 4,474 acute care hospitals had an IT supplement response rate of $62.4 \%$ ( 2,796 out of 4,474 hospitals). Using this method resulted in a loss of data for 1,425 hospitals for analysis.

This research gathered comprehensive data utilising publicly reported information which was not reliant on voluntary survey responses and included a CMS generated standardised cost per discharge metric never used before. Therefore, the results utilise a uniform lens with which to determine how EHR with MU capabilities impact cost, quality and safety. Hospital risk adjusted, outcome data and categorical variables were included into the master dataset that resulted in over 287,000 data elements for analysis. They were then divided into MU and non-MU status. This robust database combined with 
the risk adjustment scheme for the outcome indicators supported a unique and detailed statistical analysis of the difference in performance between MU and non-MU hospitals.

Two cohorts were created from the Center for Medicare and Medicaid Services (CMS) hospital database. One cohort represented hospitals that had not attested to the MU adoption of certified EHR technology as of 2013. The second cohort were hospitals that had attested to MU. For this study, data on EHR adoption followed strict inclusion criteria for meeting MU adoption standards promulgated by the ONC. The actual records were drawn from the CMS payment documentation file that records which hospitals received MU payments and in what years it was paid. Therefore, there was no need for proxy mapping (Appari et al., 2012; Furukawa et al., 2010) to interpret hospital survey responses previously required in other studies to estimate whether existing EHR met MU core standards as prescribed by the ONC.

This study utilised MU payment as the inclusion criteria for EHR adoption with MU standards. To receive MU payments, hospitals had to meet the predetermined ONC performance criteria and then 'attest' to the technology adoption. Through December 2015 , federal payments of $\$ 21$ billion have been paid to all eligible providers with nearly $\$ 13$ billion going to acute care hospital (https:/www.cms.gov/Regulations-andGuidance/ Legislation/EHRIncentivePrograms/Downloads/December2015MedicareEHRIncentiveP ayments.pdf).

Table 1 Study variables

\begin{tabular}{lcc}
\hline Variable & Measurement & Type \\
\hline EHR status & MU vs. non-MU & Independent variable \\
Total discharges & Number cases & Categorical \\
Acuity & Risk adjusted case mix index rate & Categorical \\
Teaching status & Yes or no & Categorical \\
Ownership type & Government, proprietary, voluntary (coded 1-3) & Categorical \\
Region & Midwest, northeast, south, west (coded 1-4) & Categorical \\
\hline
\end{tabular}

After the application of exclusion criteria, data was assembled on 4,221 hospitals, the performance year selected for study was 2013. There were 560 hospitals, mostly critical access facilities under 50 beds, eliminated from the study because they had less than 50 discharges per year. For facilities that had less than 30 discharges in any one of the three clinical categories that condition was excluded. The frequencies associated with the categorical variables are described in Table 2.

The profile of a hospital is comprised of demographic and operational characteristics. These characteristics or endogenous variables (Appari et al., 2012; http://www.cms. gov.Research-Statistics-Data-and-Systems/Statistics-Trends-and Reports/NationalHealth ExpendData/Downloads/highlights.pdf; Lin et al., 2015) were identified for each hospital included in the analysis shown in Table 1. The variables include hospital teaching status, hospital ownership category, and acuity of care as measured by case mix index (CMI), hospital region which was coded consistent with CMS defined regions: midwest, northeast, south and west were labelled 1-4 depending on state geography and activity level as measured by annual discharges. In Table 2 the teaching status, region and ownership are illustrated for each of the characteristics in the respective cohorts, MU and non-MU. 
Table 2 Frequencies and percentage of total by categorical variables

\begin{tabular}{lccc}
\hline Characteristic & $\begin{array}{c}\text { All hospitals } n \\
\text { (weighted \%) }\end{array}$ & $\begin{array}{c}\text { MU hospitals } n \\
\text { (weighted \%) }\end{array}$ & $\begin{array}{c}\text { Non-MU hospitals } n \\
\text { (weighted \%) }\end{array}$ \\
\hline Teaching status & $3,127(74.1)$ & $1,637(70.7)$ & $1,490(78.2)$ \\
No & $1,094(25.9)$ & $678(29.3)$ & $416(21.8)$ \\
Yes & $1,239(29.4)$ & $666(28.8)$ & $573(30.1)$ \\
Region & $575(13.6)$ & $361(15.6)$ & $214(11.2)$ \\
Midwest & $1,579(37.4)$ & $915(39.5)$ & $664(34.8)$ \\
Northeast & $780(18.5)$ & $373(16.1)$ & $407(21.4)$ \\
South & & & $479(20.7)$ \\
West & $479(20.7)$ & $444(23.3)$ & $11(0.5)$ \\
Owner & $11(0.5)$ & $28(1.5)$ & $285(15.0)$ \\
Government & $697(16.5)$ & $412(17.8)$ & $1,141(59.9)$ \\
Physician & $2,547(60.3)$ & $1,406(60.7)$ & \\
Proprietary & & & \\
Voluntary & & &
\end{tabular}

\subsection{Results}

As demonstrated in Table 3, there was a performance difference between MU and non-MU hospitals, with MU hospitals demonstrating lower cost per discharge of $\$ 327$ ( $\mathrm{p}<0.05$ ). Extrapolating this to total Medicare discharges the HITECH Act investment had a return of over $\$ 6$ billion in the first year of adoption in lowered cost of care. Prior studies have utilised various methods for detecting the impact of an EHR on hospital costs (Himmelstein et al., 2009; DesRoches et al., 2010; Appari et al., 2012; Agha, 2014; Ding and Xiaoseng, 2011; Jones et al., 2014). In these past studies, multiple approaches and data sources were utilised, some overlapping, others unique, to assess if EHR adoption had an impact on healthcare costs. The lack of consistent measures limits the external validity of these studies in the cost domain.

The difference from this research study is that none of the prior research utilised a normalised cost per discharge approach. The current study used the CMS spending by claim file (https://data.medicare.gov/Hospital-Compare/Medicare-Hospital-Spending-byClaim/nrth-mfg3/data) that calculates a normalised Medicare spending per discharge by hospital. The multiple characteristics of a hospital's overall cost structure, union versus non-union staff, urban versus rural, ownership models, payer mix and teaching status create such variability that without an adjusted cost per discharge approach there can be no meaningful cost comparison between the cohorts, MU and non-MU hospitals. By utilising a standardised metric, cost per discharge, this barrier to cost analysis has been removed in this study. The same standardised metric approach was utilised to assess the final and critically important safety domain.

Mortality outcomes similarly demonstrated a favourable difference between MU and non-MU hospitals. One of the most significant aspects of this study is that the clinical outcomes reported reveal statistically significant difference in mortality rates in all three clinical conditions $(p<0.05)$, heart attack, heart failure and pneumonia for hospitals who 
achieved MU recognition for their EHR systems. This critical finding represents both a quality and reimbursement benefit to hospitals. Based upon the difference in mortality rates an estimated 21,000 deaths were avoided.

There was a difference in favour of the non-MU hospital $(\mathrm{P}<0.048)$ across all three clinical conditions for readmission rates. The patient was categorised as readmission if they were reamitted within 30 days from the index admission with any diagnosis. This measurement is known as all cause readmission rate and is how CMS calculates the metric. The all-cause methodology for calculating this metric does confound in some respect the impact that MU technology may have on the outcome. As phase $2 \mathrm{MU}$ requirements focus more interoperability between hospitals and outside providers such as pharmacies, nursing homes, etc., greater impact on this metric may be observed at a later date.

Table 3 T-test results on quality variables by MU status

\begin{tabular}{lcccccc}
\hline $\begin{array}{l}\text { Conditions/ } \\
\text { meaningful use }\end{array}$ & & & & & \multicolumn{2}{c}{$\begin{array}{c}\text { T-test for equality } \\
\text { of means (one-tail) }\end{array}$} \\
\hline Heart attack & & $n$ & Mean & S.D. & & $d f$ \\
mortality rate ${ }^{++}$ & No & 923 & 15.210 & 1.454 & $1.735^{*}$ & 2,508 \\
Heart failure & No & 1,587 & 15.105 & 1.467 & & \\
mortality rate & 1,499 & 11.896 & 1.553 & $2.776^{*}$ & 3,623 \\
Pneumonia & Yes & 2,126 & 11.750 & 1.576 & & \\
mortality rate $^{++}$ & No & 1,671 & 12.137 & 1.856 & $3.383^{*}$ & 3,886 \\
Heart attack & Yes & 2,217 & 11.935 & 1.842 & & \\
readmit rate $^{+}$ & No & 812 & 18.232 & 1.164 & $-2.293^{*}$ & 2,236 \\
Heart failure & Yes & 1,426 & 18.352 & 1.199 & & \\
readmit rate $^{++}$ & No & 1,538 & 23.005 & 1.705 & $-1.644^{*}$ & $3,469.4$ \\
Pneumonia & Yes & 2,156 & 23.104 & 1.859 & & \\
readmit rate $^{++}$ & No & 1,679 & 17.544 & 1.344 & $-2.602^{*}$ & $3,738.9$ \\
Safety $^{++}$ & Yes & 2,221 & 17.662 & 1.448 & & \\
& No & 1,203 & 0.597 & 0.131 & $-0.116^{*}$ & $2,722.4$ \\
Cost per $_{\text {discharge }^{++}}$ & Yes & 1,960 & 0.598 & 0.143 & & \\
\hline No & 1,179 & $8,179.470$ & $2,138.442$ & $4.544^{*}$ & $1,960.3$ \\
\hline
\end{tabular}

Notes: ${ }^{+}$Equal variances not assumed for the t-test, ${ }^{++}$equal variances assumed for the t-test and *statistically significant $\mathrm{p}<0.05$.

For AHRQ composite safety score, 3,163 hospitals reported data. As illustrated in Table 3, there were 1,960 MU hospitals reporting an overall score of 0.60 and 1,203 non-MU hospitals reporting an identical score of 0.60 . There was no statistically significant difference between the MU and non-MU hospital outcomes. The limitations of using this composite safety metric are described later in the discussion section.

By analysing the actual 2013 MU attestation data file, the current study found that the percentage of hospitals that had actual MU certified EHRs' had jumped from $8 \%$ to $55 \%$, or 2,315 of 4,221 hospitals nationally. In contrast to the Desroches et al. (2013) study, the majority of hospitals that had actual MU certified functionality, 1,637 of the 2,315, or 
$71 \%$ of the attesting cohort, were actually non-teaching facilities. The majority were $(40 \%)$ located in the south, followed by the midwest, $29 \%$, west and northeast at $16 \%$ each. The predominant ownership model was $61 \%$ voluntary, not for profit status, followed by governmental $23 \%$, proprietary $18 \%$ and physician owned $1.5 \%$. The difference in the data reported by Desroches et al. (2013) and the actual CMS data results just one year later may be interpreted in several ways.

One reason for the difference in actual versus reported uptake in MU certified EHRs is that the Desroches et al. (2013) study relied on voluntary survey data with a $61 \%$ response rate. A large number of hospitals, over 1,500, did not reply, many of whom were likely not AHA members or have seen the value in completing the survey. To receive MU payments it was mandatory for hospitals to attest and to be certified as MU compliant, therefore the CMS data file used for the current study had the most current and accurate data. The other reason, also aligned with a payment incentive, is that hospitals clearly moved very quickly, a seven fold uptake, to advance their basic EHR capability once the MU criteria was finalised in order to capitalise on the HITECH funds. Therefore the financial incentive seemed to have clearly increased the number of EHR installations by those meeting the criteria. In the first year over $\$ 6$ billion was awarded to hospitals.

Table 4 Levene's test

\begin{tabular}{lcc}
\hline \multirow{2}{*}{ Quality variables } & \multicolumn{2}{c}{ Levene's test for equality of variances } \\
\cline { 2 - 3 } & $F$ & Sig. \\
\hline Heart attack readmit rate & 0.939 & $0.333^{*}$ \\
Heart attack mortality rate & 0.31 & $0.578^{*}$ \\
Heart failure readmit rate & 17.484 & $0.000^{\wedge}$ \\
Heart failure mortality rate & 1.771 & $0.183^{*}$ \\
Pneumonia readmit rate & 9.83 & $0.002^{\wedge}$ \\
Pneumonia mortality rate & 0.181 & $0.670^{*}$ \\
Safety & 9.093 & $0.003^{\wedge}$ \\
Cost per discharge & 45.419 & $0.000^{\wedge}$ \\
\hline
\end{tabular}

Notes: *Equality of variance assumed and ^equality of variance rejected.

\subsection{Limitations}

This study has several limitations. Due to the large number of hospitals, 4,221, resulting in wide geographic dispersion and other disparate attributes, the subject hospitals vary widely in numerous ways: teaching status, urban vs. rural, large vs. small discharge volume, union status and related social determinants of patients. This is a potential threat to the generalisability of the study conclusions. To control for this threat, all of the outcome measures selected were risk adjusted thereby normalising the performance and cost values across hospitals.

The data utilised for this study was abstracted from sources that utilised administrative data. As discussed above there are inherent limitations to this data source, however, outcome measures such as mortality, readmission and infection are discrete events. By focusing on mortality and readmission as quality endpoints, consistent with 
new VBP measures, this study was able to report results less subject to such data management concerns. In addition, the $3 \mathrm{M}$ risk adjustment methodology applied by CMS for the clinical outcome measures while the current standard for risk adjustment in the industry is subject to the criticism of all such formulas (Shahian and Normand, 2008). The standardised cost metric utilised for assessing cost per discharge was developed by CMS. The cost is calculated from hospital specific data and then risk adjusted for a number of variables, medical education, geographic cost allowances, etc., affecting a hospital's operating expense.

\section{Discussion}

\subsection{Impact of the HITECH Act and MU designation}

The research reported in this study utilised the database of actual MU achievement that ONC utilised for awarding MU status and distribution of payments. All of the prior studies mentioned utilised proxy measures based on either a health information management services society (HIMSS) or AHA information technology voluntary survey instrument to establish if a hospital had implemented an EHR that was capable of meeting MU specifications. These studies relied on self-reported capabilities from either of the HIMSS or AHA hospital surveys. As previously mentioned above in any given survey response year a large number of hospitals, over 1,500, did not reply omitting a significant number of organisations from their analysis. The impact of the inclusion of many non-surveyed hospitals in the current study significantly affected the true total of organisations meeting MU standards in the first two years, and affected how the cohort performed in the cost savings described below.

The implementation of basic EHRs' in the nation's hospitals stood at just $8 \%$ in 2008 (Jha et al., 2009). With the passage of the HITECH Act in 2009, ushering in both monetary incentives and penalties for EHR adoption that had to meet MU standards, a veritable rush for implementation impacted the healthcare industry. In fact by the time the first incentive payments were available in 2010-2011 period there was an over threefold increase of EHR adoption to nearly $27 \%$ of hospitals (Desroches et al., 2013). The initial uptake, according to the 2012 American Hospital Association annual IT survey, was in large, urban and teaching hospitals. The research suggested that this hospital cohort, large, urban, teaching hospitals, was twice as likely to have adopted an EHR and that approximately 44\% could meet MU standards (Desroches et al., 2013). The AHA survey response rate at just over $60 \%$ did not include the many hospitals, at least 1,500 , and the actual results from this study discussed below differed materially.

\subsection{Clinical quality}

As previously stated, one of the most significant aspects of this study is that the mortality reduction in all three clinical conditions $(\mathrm{p}<0.05)$, heart attack, heart failure and pneumonia for hospitals who achieved MU recognition for their EHR systems. This critical finding represents both a quality and reimbursement benefit to hospitals. Based upon the difference in mortality rates an estimated 21,000 deaths were avoided. The pressure to move away from fee for service reimbursement to value, driven by the VBP 
model has refocused the quality discussion to one centred on clinical outcomes and away from process measures. Previous research (Himmelstein et al., 2009; Chaudry et al., 2006; Appari et al., 2012; Ding and Xiaoseng, 2011; Spencer et al., 2010) did not demonstrate this level of clinical improvement either in magnitude of change or uniformly across conditions. It is important to discuss the focus on process versus outcome as a measurement paradigm in this prior research to understand the different outcomes of the studies.

There are numerous hypotheses as to why hospitals with MU certified technology have lower mortality (Shekelle et al., 2011; Bates et al., 2001). The adoption of evidence-based guidelines, prompt response to changes in lab and physiologic data, automated medication management for drug-drug interaction, automated alerts to changes in lab values, etc. What is important to note is that by using risk adjusted data the outcomes have statistical control for the influence of variables such as CMI, teaching vs. non-teaching status, urban vs. rural and other confounding factors associated with mortality (Table 2).

The focus on population health inherent to VBP requires a measurement lens of broader scope and one oriented to informing wider aspects of health policy (Mant, 2001) that being outcomes. Outcome measures such as mortality, readmission and infection are discrete events. By focusing on mortality and readmission as quality endpoints, consistent with new VBP measures, this current study was able to report results less subject to such data management concerns (Mant, 2001).

\subsection{Cost}

The fact that a statistically significant difference in mean cost per Medicare discharge between MU and non-MU hospitals of $\$ 327$ was found in this study provides support for the $\$ 50$ billion investment of the HITECH Act. In one year, with just over $50 \%$ of the nation's hospitals participating, the current study suggests over $\$ 6$ billion in savings will have accrued from MU adoption. The focus on whether EHRs' demonstrated a relationship to cost in the $\$ 3$ trillion US healthcare system was a significant focus on this study. CMS sought to bend the cost curve and align payment with value by implementing VBP and shifting the original reimbursement equation weighted $90 \%$ in favour of process to the current 2016 formula which is $90 \%$ outcome oriented (https://www.medicare.gov/ hospitalcompare/data/total-performance-scores.html).

\subsection{Safety}

The previous research on EHR impact on safety outcomes focused on specific initiatives in local hospital or health system (Poon et al., 2010). The seminal study To Err is Human (Institute of Medicine, 1999) identifying between 44,000 to 98,000 deaths annually from errors was followed by numerous other studies (Poon et al., 2010; Institute of Medicine, 1999; Shekelle et al., 2011; Bates et al., 2001). Some recent studies actually found that EHR created its own error prone process problems and a caution flag was raised (Sittig and Singh, 2012; Institute of Medicine, 2011). Few if any broad-based studies were focused on this topic because of the complexity in measuring and identifying agreed upon community standard for analysis. The Agency for Health Care Research and Quality (Barclay et al., 2019) safety metric PSI 90, changed this barrier. 
Unfortunately, the indicators selected for the composite score are not well aligned with clinical interventions that EHR can specifically impact. There are 11 indicators, nine of which are surgery related, followed by pressure ulcers and blood stream infection. In order to better assess the impact of EHR on patient safety a different indicator set more effective in impacting safety issues such as medication errors, timing of antibiotic for procedures, pneumonia care or management of sepsis would be more sensitive measures (Poon et al., 2010; Bates et al., 2001).

\subsection{Conclusions}

Utilising a revised measures paradigm, one focused on publicly reported outcome measures, not process indicators, the study found a statistically significant correlation between MU adoption of certified electronic health records and reduced cost per discharge and lower mortality in the Medicare population. This study estimated the mortality difference achieved at MU hospitals resulted in 21,000 fewer in-patient deaths. This is a powerful finding and one further magnified when taken in the context that over $\$ 6$ billion in cost per discharge was achieved by the same cohort, MU hospitals. The results will have profound interest among patients, providers as well as commercial and governmental healthcare payers.

\subsection{Implications}

These results are the first definitive endorsement of MU capability in clinical quality and cost savings. When considering that the lead time for adopting complex technology is estimated between two and five years (Porter, 2006), the fact that mortality differences and cost savings were demonstrated in the first two years after the initial attestation period, 2013, supports the CMS investment in MU subsidy. Hospitals continue to be subject to the CMS value-based purchasing rules (https://www.cms.gov/Medicare/ Quality-Initiatives-Patient-Assessment-Instruments/HospitalQualityInits/Hospital-ValueBased-Purchasing-.html) which withhold $2 \%$ of Medicare payments. To earn these funds and any potential bonus available, hospitals must achieve quality metrics of which mortality, cost and complications are substantial component. The effective use of EHR technology is an important tool for this goal.

\section{Acknowledgements}

The authors would like to acknowledge Ms. Val Lajqi, MBA for the many hours of invaluable support in proofing and preparing the manuscript and Mr. Nick Szymanski for superior technical effort in the database development. Dr. Conte would like to recognise Dr. Susan Carroll for her assistance in the theoretical and measurement framework development.

\section{References}

Agha, L. (2014) 'The effects of health information technology on the costs and quality of medical care', Journal of Health Economics, Vol. 34, No. C, pp.19-30, Job Market Paper. 
Appari, A., Eric Johnson, M. and Anthony, D.L. (2012) 'Meaningful use of electronic health record systems and process quality of care: evidence from a panel data analysis of U.S. acute-care hospitals', Health Services Research, DOI: 10.1111/j.1475-6773.2012.01448.x.

Barclay, M., Dixon-Woods, M. and Lyratzopoulos, G. (2019) 'The problem with composite indicators', BMJ Quality \& Safety, Vol. 28, pp.338-344.

Bates, D., Cohen, M., Leape, L. et al. (2001) 'Reducing the frequency of errors in medicine using information technology', Journal of the American Medical Informatics Association, Vol. 8, No. 4, pp.299-308.

Blumenthal, D. and Tavenner, M. (2010) 'The 'meaningful use' regulation for electronic health records', The New England Journal of Medicine, Vol. 363, No. 6, pp.501-504.

Bunti, M., Burke, M., Hoaglin, M. et al. (2011) 'The benefits of health information technology: a review of the recent literature shows. Predominantly positive results', Health Affairs, Vol. 30, No. 3, pp.464-474.

Chaudry, B., Wang, J., Wu, S. et al. (2006) 'Systematic review: impact of health information technology on quality, efficiency, and costs of medical care', Annals of Internal Medicine, Vol. 144, No. 10, pp.742-751.

DesRoches, C., Campbell, E., Vogeli, C. et al. (2010) 'Electronic health records limited successes suggest more targeted uses', Health Affairs, Vol. 29, No. 4, pp.639-646.

Desroches, C., Charles, D., Furukawa, M. et al. (2013) 'Adoption of electronic health records grows rapidly, but fewer than half of U.S. hospitals had at least a basic system in 2012', Health Affairs, Vol. 32, No. 8, pp.1478-1484.

Ding, D. and Xiaoseng, D. (2011) 'Long-term effects of electronic medical records on hospital clinical, financial and operational performance', POMS Conference Proceedings, Abstract Number: 025-0837.

Donabedian, A. (1998) 'The quality of care how can it be measured?', Journal of American Medical Association, Vol. 260, No. 12, pp.1743-1748.

Donabedian, A. (2005) 'Evaluating the quality of medical care', The Milbank Quarterly, Vol. 83, No. 4, pp.691-729.

Furukawa, M., Raghu, T. and Shao, B. (2010) 'Electronic medical records and cost efficiency in hospital medical-surgical units', The Journal of Health Care Organization, Provision, and Financing, Vol. 47, No. 2, 110-123 [online] https://doi.org/10.5034/inquiryjrnl_47.02.110.

Hillestad, R., Bigelow, J., Bower, A. et al. (2005) 'Can electrocix medical record systems transform health care? Potential health benefits, savings, and costs', Health Affairs, Vol. 24, No. 5, pp.1103-1117.

Himmelstein, D., Wright, A. and Woolhandler, S. (2009) 'Hospital computing and the costs and quality of care: a national study', The American Journal of Medicine, Vol. 123, No. 1, pp.1-7.

Institute of Medicine (1999) To Err is Human: Building a Safer Health System, Author, Washington DC.

Institute of Medicine (2001) Crossing the Quality Chasm: A New Health System for the 21st Century, The National Academies Press, Washington, DC [online] https://doi.org/10.17226/10027.

Institute of Medicine (2011) IOM Weighs in on EHR Safety, Contributing Writer.

Jha, A., DesRoches, C., Campbell, E. et al. (2009) 'Use of electronic health records in U.S. hospitals', N. Engl. J. Med., Vol. 360, No. 16, pp.1628-1638.

Jones, S., Rudin, R., Perry, T. et al. (2014) 'Health information technology: an updated systematic review with a focus on meaningful use', Annuals of Internal Medicine, Vol. 160, No. 1, pp.48-54.

Lin, Y., Lin, M. and Chen, H. (2015) Does Meaningful Use of Electronic Health Records Improve Quality of Care?, 11 July [online] SSRN: https://ssrn.com/abstract=2444054; http://dx.doi.org/10.2139/ssrn.2444054. 
Mant, J. (2001) 'Process versus outcome indicators in the assessment of quality health care', International Journal for Quality in Health Care, Vol. 13, No. 6, pp.475-480.

McHugh, M. and Joshi, M. (2010) 'Improving evaluations of value-based purchasing programs', Health Services Research, Vol. 45, No. 5, pp.1559-1569.

Poon, E., Keohane, C., Yoon, C. et al. (2010) 'Effect of bar-code technology on the safety of medication administration', New England Journal of Medicine, Vol. 362, No. 18, pp.1698-1707.

Porter, M. (2006) Redefining Healthcare: Creating Value-Based Competition on Results, pp.1-385, Harvard Business School Press, Boston, MA.

Ranawat, A., Nunley, R. and Bozic, K. (2009) 'Value-based purchasing and technology assessment in orthopaedics', Clinical Orthopaedics and Related Research, Vol. 467, No. 10, pp.2556-2560.

Shahian, D. and Normand, S. (2008) 'Comparison of 'risk-adjusted' hospital outcomes', Circulation, Vol. 17, pp.1955-1963.

Shekelle, P., Pronovost, P., Wachter, R. et al. (2011) 'Advancing the science of patient safety', Annuals of Internal Medicine. Vol. 154, No. 10, pp.693-696, Coordinator for Health Information Technology.

Sittig, D. and Singh, H. (2012) 'Electronic health records and national patient-safety goals', The New England Journal of Medicine, Vol. 367, No. 19, pp.1854-1860.

Spencer, J., Adams, J., Schneider, E., Ringel, J. et al. (2010) 'Electronic health record adoption and quality improvement in US hospitals', American Journal of Managed Care, Vol. 16, No. 12, SP-64-SP71.

VanLare, J. and Conway, P. (2012) 'Value-based purchasing - national programs to move from volume to value', The New England Journal of Medicine, Vol. 367, No. 4, pp.292-295.

\section{Websites}

http://www.cms.gov.Research-Statistics-Data-and-Systems/Statistics-Trends-andReports/ NationalHealthExpendData/Downloads/highlights.pdf.

https://www.cms.gov/Medicare/Quality-Initiatives-Patient-Assessment-Instruments/

HospitalQualityInits/Hospital-Value-Based-Purchasing-.html. 